Military Technical College Kobry El-Kobbah, Cairo, Egypt

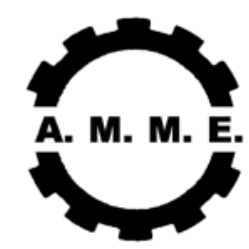

\section{$14^{\text {th }}$ International Conference on Applied Mechanics and Mechanical Engineering AMME-14}

\title{
Phase field simulation of ferroelectrics with defect
}

By

X.F. Zhao*

A.K. Soh*

\section{Abstract:}

By employing a dipole defect model, two-dimensional phase field simulations of domain switching in the crack tip vicinity of a crack embedded in a relaxor ferroelectric single crystal subjected to mechanical loading were carried out. The interaction between the dipole defects and crack, and the influence of the dipole defect concentration density on the switching process has been investigated. Comparison of the results obtained from normal and relaxor ferroelectrics showed that for the latter the polarization switching in the vicinity of the crack tip was suppressed and the field distribution was not symmetric to the crack surface, which was different from those of the former. These were due to the interaction between the dipole defects and crack, and the inhomogeneous field induced by dipole defects. Moreover, the polarization switching rate and switching zone area decreased with the increase of dipole defect concentration density.

\section{Keywords:}

Normal ferroelectric, relaxor ferroelectric, phase field simulation, domain switching, crack

* Department of Mechanical Engineering, The University of Hong Kong, Pokfulam Road, Hong Kong, China 


\section{Introduction:}

In recent years, the relaxor ferroelectrics (RFs) have been of a focus owing to their exceptional dielectric and electromechanical behaviours compared to normal ferroelectrics (NFs) over a broad temperature range. A typical RF displays a diffuse phase transition, a strong frequency dispersion of the dielectric properties, and an absence of macroscopic polarization at zero electric field[1].

Since the origin of RF's dielectric behaviour has yet to be completely understood, various models have been proposed[2]. Among the models, the dipole defect model is an important one, in which dipole defects are introduced into a NF material, resulting in internal random fields with respect to the local dipole moment and a change in the stability of the local ferroelectric order[3]. By employing Monte Carlo simulations and Ginzburg-Landau theory, Su et al. [4] identified dipole clustering above the ferroelectric phase transition temperature and cluster freezing far below this temperature. Liu et al. [2] have studied the influence of dipole defect concentration and temperature on dipole configuration and dielectric susceptibility of RFs. Li and Liu [5] have investigated the intrinsic correspondence between dipole-clustering/freezing and the multi-peaked timedomain distribution of dielectric relaxation. Wang and Liu [3] have shown that the role of long-range elastic energy in RFs was much less important than that in NFs.

A great number of theoretical and experimental studies have been carried out to investigate the properties of normal and RF materials under electric-mechanical loading. Among these, Soh et al. [6] simulated the dielectric and elastic response to electromechanical loading. However, owing to the intrinsic brittleness of ferroelectrics, microcracks can easily form and accumulate in the materials. Therefore, a better understanding of the fracture behaviour of RFs is of great importance in improving the design of components made of such materials. In the work of Song et al. [7], in which crack tip domain switching was simulated in a ferroelectric material, polarization switching regions were found at the crack tip.

In the present paper, by employing the time-dependent Ginzburg-Landau theory and phase-field method (PFM), the polarization switching process near the tip of a crack in a RF subjected to tension perpendicular to the crack surface was simulated, and the interaction of dipole defect and crack on the domain switching was discussed.

\section{Modle and Simulation:}

In the present paper, the domain evolution of RF under the application of mechanical loading is studied, and the relaxor is modelled by including spatially distributed dipole defects in a NF material.

The total free energy of a relaxor can be established based on that of the NF by adjusting the energy coefficients. Based on Ginzburg-Landau-Devonshire theory, the 
total free energy $F$ for a NF system consists of a bulk free energy $F_{l}$, a gradient energy $F_{g}$, a dipole-dipole interaction energy $F_{\text {dip }}$, an electrostatic energy $F_{e s}$, and an elastic energy $F_{\text {ela }}$. It was pointed out in ref. [3] that for RFs, the long-range ordering is broken with doping by dipole defects and the elastic energy stored inside dipole clusters may be effectively released by the surrounding paraelectric matrix. Thus, in the present study, the electrostrictive energy term is not considered; while the elastic energy term is kept owing to the presence of a crack, which is considered as an eigenstrain in the following simulation.

Assuming that the boundary conditions are periodic, for a cubic system, the bulk free energy density $f_{1}$ can be expressed as follows:

$$
f_{l}\left(P_{i}\right)=A_{1}\left(P_{x}^{2}+P_{y}^{2}\right)+A_{11}\left(P_{x}^{4}+P_{y}^{4}\right)+A_{12} P_{x}^{2} P_{y}^{2}+A_{111}\left(P_{x}^{6}+P_{y}^{6}\right)+A_{112}\left(P_{x}^{4} P_{y}^{2}+P_{x}^{2} P_{y}^{4}\right)(1)
$$

where $P_{i}(\boldsymbol{r})$ is the $i$ th component of the moment vector $\boldsymbol{P}(\boldsymbol{r})$ at site $\boldsymbol{r}$; $A_{1}=\frac{T-T_{0}}{2 \varepsilon_{0} C_{0}}=\frac{1}{2 \varepsilon_{0} \chi}$, in which $\varepsilon_{0}, \chi, T, T_{0}$ and $C_{0}$ denote the vacuum permittivity, the dielectric susceptibility of the material, the temperature, the Curie-Weiss temperature and the Curie constant, respectively.

Liu et al. [2] has illustrated that the stability of a local dipole will be changed by introducing defects into the lattice. Thus, the moment of a dipole at the site with a defect attached can be enhanced or suppressed. Since the stability of a dipole is mainly dependent on the coefficient $A_{1}$ in the expression of the bulk free energy, the other coefficients in the said energy are assumed to be independent of the dipole sites [7]. That is,

$$
A_{1}(\boldsymbol{r})=A_{10}+b_{m} \cdot c(r)
$$

The parameter $c$ of value between 0 and 1 labels the defect state of a site; if $c=0$, the material at site $\boldsymbol{r}$ is perfect, and the other extreme, i.e., $c=1$, means that a defect is imposed at this site. The parameter $b_{m}$ is the coefficient characterizing the influence of defects on the dipole stability. A positive or negative value of $b_{m}$ indicates a suppression or enhancement function, respectively, on the instability of the dipole. Note that in our simulation, the RF is assumed to be at room temperature, and the influence of temperature is neglected.

The gradient energy density $f_{g}$ is given by: 
$f_{g}\left(P_{i, j}\right)=\frac{1}{2} G_{11}\left(P_{x, x}^{2}+P_{y, y}^{2}\right)+G_{12} P_{x, x} P_{y, y}+\frac{1}{2} G_{44}\left(P_{x, y}+P_{y, x}\right)^{2}+\frac{1}{2} G_{44}^{\prime}\left(P_{x, y}-P_{y, x}\right)^{2}$

where $P_{i, j}=\frac{\partial P_{i}}{\partial r_{j}}$.

The dipole-dipole interaction energy density $f_{\text {dip }}$ is:

$$
f_{\text {dip }}\left(P_{i}\right)=\frac{1}{8 \pi \varepsilon_{0} \chi} \int d^{3} r_{j}\left\{\frac{\boldsymbol{P}\left(r_{i}\right) \boldsymbol{P}\left(r_{j}\right)}{\left|r_{i}-r_{j}\right|^{3}}-\frac{3\left[\boldsymbol{P}\left(r_{i}\right)\left(r_{i}-r_{j}\right)\right]\left[\boldsymbol{P}\left(r_{j}\right)\left(r_{i}-r_{j}\right)\right]}{\left|r_{i}-r_{j}\right|^{5}}\right\}
$$

The electrostatic energy density $f_{e s}$ is:

$$
f_{e s}\left(P_{i}\right)=-P_{i} E_{i}
$$

The elastic energy density is expressed as

$$
f_{e l a}\left(u_{i, j}\right)=\frac{1}{2} C_{i j k l}^{0}\left(\varepsilon_{i j}-\varepsilon_{i j}^{0}\right)\left(\varepsilon_{k l}-\varepsilon_{k l}^{0}\right)=\frac{1}{2} C_{11}\left(\eta_{x x}^{2}+\eta_{y y}^{2}\right)+C_{12} \eta_{x x} \eta_{y y}+2 C_{44} \eta_{x y}^{2}(6)
$$

In Eqn. (6), $\eta_{i j}=\varepsilon_{i j}-\varepsilon_{i j}^{0}$ denotes the elastic strain, and $\varepsilon_{i j}^{0}$ stands for eigenstrain. The eigenstrains resulted from polarization is denoted as $\varepsilon_{i j}^{p}$, which can be expressed as:

$$
\begin{aligned}
& \varepsilon_{11}^{p}=Q_{11} P_{x}^{2}+Q_{12} P_{y}^{2} ; \\
& \varepsilon_{22}^{p}=Q_{11} P_{y}^{2}+Q_{12} P_{x}^{2} ; \\
& \varepsilon_{12}^{p}=Q_{44} P_{x} P_{y} .
\end{aligned}
$$

where $Q_{11}, Q_{12}, Q_{44}$ are the electrostrictive energy coefficients. By employing the method used by Jin et al. [9], the influence of crack on the mechanical system of the structure can be treated as an eigenstrain in the crack area $\varepsilon_{i j}^{c}$. Therefore, the sum of the eigenstrains is given by:

$$
\varepsilon_{i j}^{0}(\boldsymbol{r})=\varepsilon_{i j}^{p}(\boldsymbol{r})+\varepsilon_{i j}^{c}(\boldsymbol{r})
$$

In the present study, the moment vector $P_{i}(\boldsymbol{r}, t)$ and eigenstrain $\varepsilon_{i j}^{c}$ in the crack area are 
set as order parameters; thus, the governing Ginzburg-Landau (TDGL) equations are expressed as follows:

$$
\begin{aligned}
& \frac{\partial P_{i}(\boldsymbol{r}, t)}{\partial t}=-L \frac{\delta F}{\delta P_{i}(\boldsymbol{r}, t)}+\xi_{i}(\boldsymbol{r}, t) \\
& \frac{\partial \varepsilon_{i j}^{c}(\boldsymbol{r}, t)}{\partial t}=-L \frac{\delta F}{\delta \varepsilon_{i j}^{c}(\boldsymbol{r}, t)}+\xi_{i}(\boldsymbol{r}, t)
\end{aligned}
$$

where $L$ is the kinetic coefficients; and tdenotes time; $\delta F / \delta P_{i}(\boldsymbol{r}, t)$ and $\delta F / \delta \varepsilon_{i j}^{c}$ represent the thermodynamic driving force for the spatial and temporal evolutions of $P_{i}(\boldsymbol{r}, t)$ and for the evolutions of eigenstrain, respectively. The kinetic coefficients for the evolution of polarization vector and eigenstrain are assumed to be the same in the simulation. Note that the noise term is neglected for simplicity in the following simulation.

\section{Simulation results and Discussion:}

It was pointed out by Ozgul [10] that, because the dipolar regions in RF are randomly oriented along different polarization direction, the crystal appears to be isotropic. Thus, the RF material is assumed to be isotropic in the following simulation. Note that $\mathrm{PbTiO}_{3}$ is chosen as the basic NF material. The main normalized material constants and parameters [7] employed in the simulation are listed in table 1.

In the present study, two-dimensional phase field simulations are carried out to investigate domain switching in the vicinity of the crack, and a normalized uniform tension stress, $\sigma_{22}^{a p p{ }^{*}}=1.3$, is applied in the vertical direction. Fig. 1 shows the geometrical configuration of the structure to be studied, in which a $128 \times 128$ lattice along both Cartesian axes is discretized for simulation. As it is pointed in the paper of Song et al [7], the length of the crack is assumed to be 24 grid interspaces, and the width of the crack is assumed to be 5 grid interspaces. The lattice spacing is chosen to be $\Delta x=1.0$. The time step is $\Delta \tau=0.01$. Assume that the original dipolar configuration is a single domain with dipole vectors along the positive $x$ direction, as shown in Fig. 1. Tension is applied along $y$ direction. It should be pointed that in the following simulation, $C_{p}$ is assumed to be 0.5 , while $C_{0}$ changes, where $C_{0}$ denotes the total dipole defect concentration density, and $C_{p}$ is the concentration density value of the dipole defect which can enhance the dipole stability.

Figure 2 shows the polar vector distribution for RF materials with $C_{0}=0.0,0.2,0.4,0.6$ at 400 time steps, and the central $64 \times 64$ domain structures are shown for the sake of 
clarity. Figure 2(a) presents the corresponding domain switching areas in NF in the vicinity of crack. The results are similar to those obtained by Song et al [7]. It can be seen from Figure 2 that under the mechanical loading, as shown in Figure 1, the domain switching of the RF forms $90^{\circ}$ domains as in the case of NF. Moreover, some phenomena can also be observed. Firstly, in all cases, switching starts in the direction of about $4^{\circ}$ with respect to the $x$ axis, which is the direction of the largest driving force. Secondly, with the increase of dipole defect concentration, the areas of the switching regions in the vicinity of the crack shrink rapidly. This indicates that the existence of the dipole defect decreases the driving force for domain switching, and such forces decreases with the increase of the dipole defect concentration. Thirdly, while the domain switching zone in NF are symmetric with respect to the crack surface and the $y$ direction, the existence of the dipole defects in RF makes the domain switching zones asymmetric with respect to both the crack surface and the $y$ direction. Moreover, the decrease of dipole defect concentration density leads to smoother switching region envelope. The above observations confirm that the existence of dipole defects is the cause of asymmetric driving force and domain switching zone in the vicinity of the crack tip. Last but not least, the increase of dipole defect concentration density leads to decreasing rate of switching in the vicinity of the crack.

Table 1: Parameters used for simulation

\begin{tabular}{|c|c|c|c|c|c|c|c|c|c|c|c|c|c|c|}
\hline$A_{11}$ & $A_{12}$ & $A_{111}$ & $A_{112}$ & $G_{11}$ & $G_{12}$ & $G_{44}$ & $G_{44}^{\prime}$ & $Q_{11}$ & $Q_{12}$ & $Q_{44}$ & $\mu$ & $v$ & $C_{p}$ & $\left|b_{m}\right|$ \\
\hline-0.24 & 2.5 & 0.49 & 1.2 & 1.6 & 0.0 & 0.8 & 0.8 & 0.05 & -0.015 & 0.019 & 481.7 & 0.3 & 0.5 & 10 \\
\hline
\end{tabular}




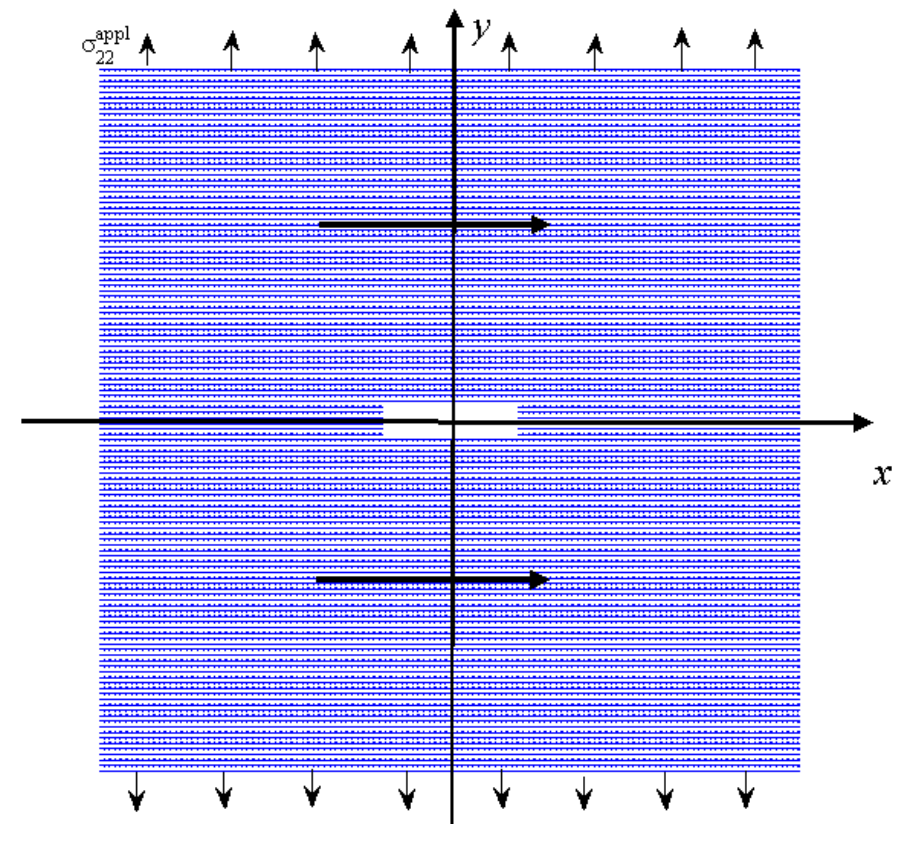

Figure 1: Parameters used for simulation 

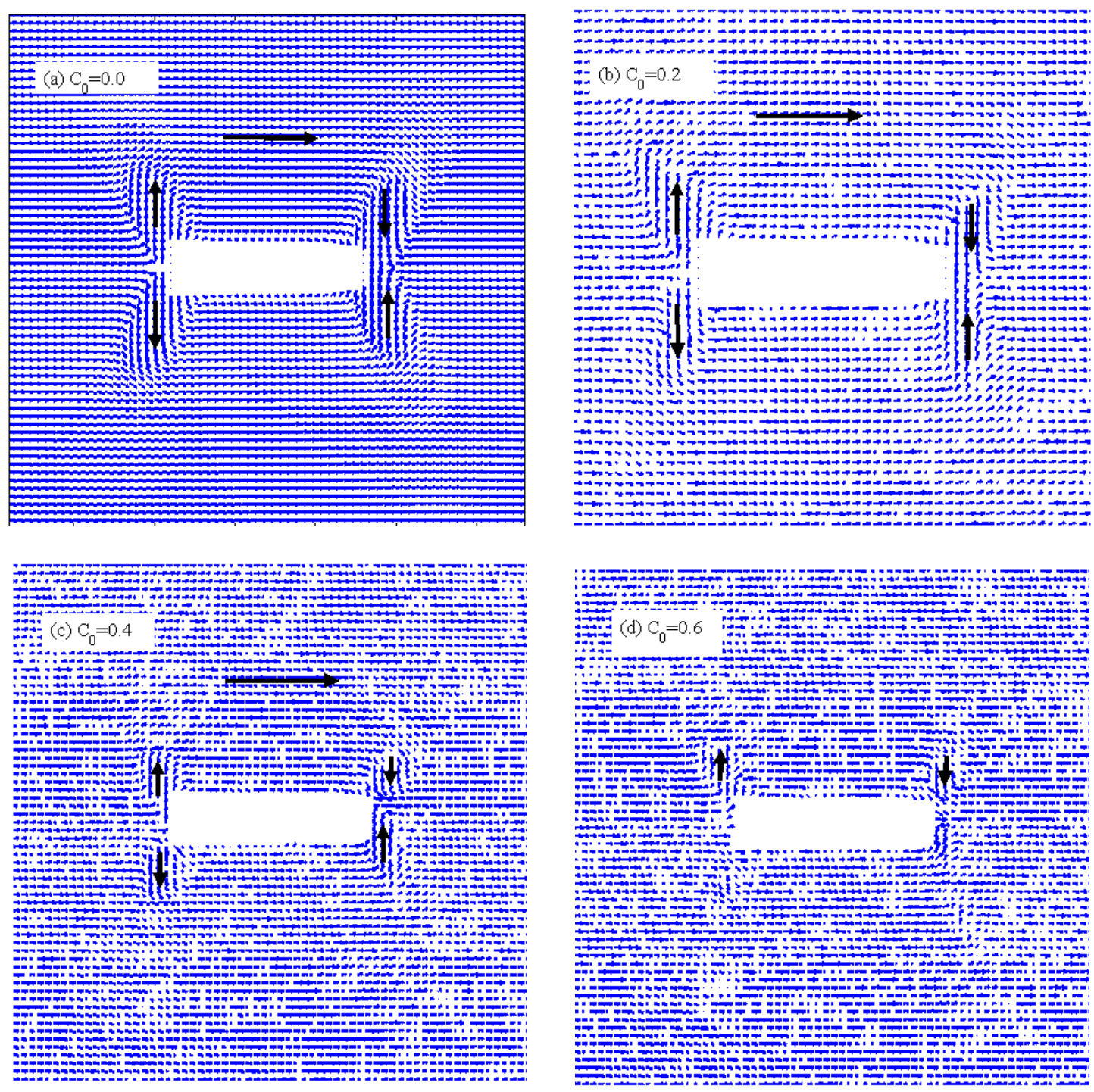

Figure 2: The evolution of domains in the vicinity of crack at 400 time steps for different $C_{0}$ value
(a) $\mathrm{C} 0=0.0$
(b) $\mathrm{C} 0=0.2$
(c) $\mathrm{C} 0=0.4$
(d) $\mathrm{C} 0=0.6$

\section{Conclusions:}

By employing Ginzburg-Landau theory and the phase-field method, simulations were carried out to study the interaction between dipole defects and the crack in a RF material subjected to remote tensile loading. The phenomena observed during the polarization switching process of RF materials are: (i) decrease of polarization switching rate and area of final switching zone with increasing dipole defect concentration density, which means that dipole defects suppress polarization switching; (ii) asymmetric polarization switching with respect to the $x$ axis in RF, which can be explained by the interaction of dipole defects and the crack giving rise to an inhomogeneous internal electric field in 
the material, and, thus, suppressing the electric field in an asymmetric manner. These phenomena are important for the design and manufacture of smart structures using RF materials.

\section{Acknowledgement:}

Support from the Research Grants Council of the Hong Kong Special Administrative Region, China (Project no. HKU 716007E) is acknowledged.

\section{References:}

[1] L. E. Cross, Relaxor Ferroelectrics, Ferroelectrics, Vol. 76, No. 3-4, P. 241-267, 1987.

[2] J. M. Liu, X. Wang, H. L. W. Chan and C. L. Choy, Monte Carlo Simulation of the Dielectric Susceptibility of Ginzburg-Landau Mode Relaxors, Physics Review B, Vol. 69, No. 9, P. 094114 1-6, March 2004.

[3] L. F. Wang and J. M. Liu, Role of Long-range Elastic Energy in Relaxor Ferroelectrics, Applied Physics Letter, Vol. 89, No. 9, P. 092909 1-3 August 2006.

[4] C. C. Su, B. Vugmeister and A. G. Khachaturyan, Dielectric Properties of Material with Random Off-center Defects: Monte Carlo Simulation of Relaxor Ferroelectrics, Journal of Applied Physics, Vol. 90, No. 12, P. 6345-6356, December 2001.

[5] Q. C. Li, and J. M. Liu, Dipole Cluster State and Dielectric Relaxation in Relaxor Ferroelectrics, Key Engineering Matererials Vol. 368-372 P. 565-569, February 2008.

[6] A. K. Soh, Y. C. Song, and Y. Ni, Phase Field Simulation of Hysteresis and Butterfly Loops in Ferroelectrics Subjected to Electro-mechanical Coupling Loading, Journal of American Ceramic Society, Vol. 89, No. 2, P. 652-661, February 2006.

[7] Y. C. Song, A. K. Soh, and Y. Ni, Phase Field Simulation of Crack Tip Domain Switching in Ferroelectrics, Journal of Physics D: Applied Physics, Vol. 40, No. 4, P. 1175-1182 February 2007.

[8] S. Semenovskaya and A. G. Khachaturyan, Development of Ferroelectric Mixed States in a Random Field of Static Defects, Journal of Applied Physics, Vol. 83, No. 10, P. 5125-5136, February 1998. 
[9] Y. M. Jin, Y. U. Wang, and A. G. Khachaturyan, Three-dimensional Phase Field Microelasticity Theory of a Multivoid Multicrack System in an Elastically Anisotropic Body: Model and Computer Simulation, Philosophical Magazine, Vol. 83, No. 13, P. 1587-1611, May 2003.

[10] M. Ozgul, Polarization Switching and Fatigue Anisotropy in Relaxor-lead titanate Ferroelectric Single Crystals, PhD Dissertation, The Pennsylvania state University 2003. 